\title{
Effect of Matter Distribution on Relativistic Time Dilation
}

\author{
Dirk J. Pons ${ }^{1 *}$, Arion D. Pons ${ }^{2}$, Aiden J. Pons ${ }^{3}$ \\ ${ }^{1}$ Department of Mechanical Engineering, University of Canterbury, Christchurch, New Zealand \\ ${ }^{2}$ University of Cambridge, Cambridge, UK \\ ${ }^{3}$ University of Canterbury, Christchurch, New Zealand \\ Email:^dirk.pons@canterbury.ac.nz, arion.pons@gmail.com, aidenpons@gmail.com
}

How to cite this paper: Pons, D.J., Pons, A.D. and Pons, A.J. (2018) Effect of Matter Distribution on Relativistic Time Dilation. Journal of Modern Physics, 9, 500-523. https://doi.org/10.4236/jmp.2018.93035

Received: January 23, 2018

Accepted: February 25, 2018

Published: February 28, 2018

Copyright (๑ 2018 by authors and Scientific Research Publishing Inc. This work is licensed under the Creative Commons Attribution International License (CC BY 4.0).

http://creativecommons.org/licenses/by/4.0/

\begin{abstract}
Context: Derivations for the relativity formulations for the Lorentz are conventionally based on continuum mechanics. Purpose: This paper derives the formulations from a particle perspective. Approach: A non-local hidden-variable (NLHV) approach is adopted, based on the specific particle structures of the Cordus Theory. Findings: The Lorentz and relativistic Doppler formulations are shown to be derivable from a NLHV particle perspective. Unexpectedly, the equations contain an additional term relating to the difference in the distribution of matter (fabric density) between situations. For a homogenous fabric, which is the assumption of general relativity, the conventional formulations are recovered. Originality: The novel contribution is deriving the relativistic formulation from a NLHV theory. Also novel is the identification of the fabric density as a term in the Lorentz. Implications: It is predicted that inertial frames of reference are only situationally equivalent in the special case where they also have the same fabric density. We find against the cosmological principle with its assumption of homogeneity. The resulting situational theory of relativity has further implications for interpreting gravitational interactions at the galactic scale and larger.
\end{abstract}

\section{Keywords}

Gravitation, Special Relativity, Cordus Theory, Non-Local Hidden-Variable

\section{Introduction}

Relativity provides the formulations for how an observer in one frame of reference perceives motion in another, and includes the phenomena of time dilation, relativity of simultaneity and the relativistic Doppler Effect. The Lorentz trans- 
formation is conventionally explained from the continuum perspective of relativity [1]. There is a long history of derivations from various perspectives [2] [3], with equifinality in outcomes. All are based on a number of postulates about the nature of measurement specifically the invariance of an interval of space [4], that space-time is a continuum [5], and that an effect is not transmitted instantaneously through space.

It is possible to derive the expression for the Lorentz factor $\gamma$ from first principles with such postulates. The Lorentz factor may then be introduced into quantum mechanics. A relativistic quantum mechanics is obtainable by substituting quantum wave functions for the classical variables, hence forming the Klein-Gordon and Dirac equations. However, the particle perspectives do not offer a derivation of the Lorentz factor from their own first principles: they use what is derived elsewhere.

Consequently, there is value in seeking to derive the Lorentz from first principles with a particle theory. Doing so has the potential to elucidate further insights into how gravitational and particle theories may be integrated. This paper derives the Lorentz transformation from a particle mechanics, specifically a non-local hidden-variable (NLHV) design. Underpinning the NLHV approach is the premise that structure exists at the sub-particle level, and that physical realism applies. Physical realism is a premise about causality: physical phenomena have deeper causal mechanics involving parameters that exist objectively.

\section{Context}

In the present work the specific particle theory area under examination is the hidden-variable sector. Historically the non-local hidden-variable (NLHV) theories have been unproductive. Non-locality has to exist if quantum superposition and entanglement are to be accepted as true (we do accept both these phenomena). Quantum superposition identifies that a particle may be in two geometric locations at once. Entanglement identifies that effects at one superposition location are superluminally communicated to the other location. However non-locality also conflicts with the general expectation that particles are zero dimensional points and that they should only be disturbed by forces, fields, or effects acting at the location of the point (not remotely). NLHV theories attempt to solve this problem by proposing that particles have sub-structure. However predicting the structure is not straight forward, and there are many possible variants. An early development was the de Broglie-Bohm Theory [6] [7]. Neither this nor the other theories that have subsequently arisen have resulted in a comprehensive theory of physics.

Nonetheless, an attractive feature of the hidden-variable sector is that it offers the potential for a theory based on physical realism [8], and hence different routes towards a physics that unifies particle and gravitational effects. A recent development in the NLHV sector is the Cordus Theory [9]. This arose from 
conceptual design and systems engineering principles applied to determine the requisite sub-structures that would be sufficient to explain the photon behaviour in the double-slit device. The result is a specific prediction of structures within the particle. From this perspective the sub-particle structure comprises two reactive ends (as opposed to a single point). These ends are some geometric separation apart and connected by a fibril. The theory requires these reactive ends to energize at a frequency, and emit discrete forces when they do. These discrete forces determine the identity of the particle (electron, neutrino, etc.), and are proposed to be connected in a flux tube. These structures are shown in Figure 1.

\section{Electron e}

Characterised by one discrete force in each of the three directions. This balanced loading causes the structure to be stable against decay. Physical structure

The discrete forces are released rather than retained as in the photon. Consequently there is an enduring succession of discrete forces in each of the three directions, which creates a long-ranged force effect.

New discrete forces continue to be created and sent down the flux tube at each frequency cycle Inner Fibril provides instantaneous communication between reactive ends, hence a non-local effect

Type of reactive end: pulsatile. One reactive end energising and the other de-energising $\left(180^{\circ}\right.$ out of phase)

\section{Notation}

The HED notation represents the distribution of the discrete forces in the three emission directions (HEDs)

Three orthogonal axes $(r, a, t)$ for emission of discrete forces

$$
e\left(r^{1} \cdot a^{1} \cdot t^{1}\right)
$$

Each discrete force carries a $1 / 3$ electrical charge, with the super/subscript representing the direction, so electron has overall -1 charge.

Figure 1. The representation of the electron's internal and external structures. It is proposed that the particle has three orthogonal discrete forces, energized in turn at each reactive end. Adapted from [10]. 
The sub-structures, e.g. those shown for the electron, are based on systematic design principles applied to determine the requisite functionality of a particle, not on arbitrary hypotheses. It is called the Cordus Theory because of its proposed linear structures. This structure is termed a particule to distinguish it from the zero-dimensional point particle of quantum mechanics. As will be shown, it is the stretch of the flux tube that is proposed as the underlying physical basis for the Lorentz.

An important component of the theory is the concept of fabric. In this context fabric is a concept in the Cordus Theory that refers to the composition of the vacuum. The theory proposes that all massy particules emit discrete forces of electric charge, and that these propagate out into the space between other massy particles. Consequently, the universe, including the vacuum of space, is filled with the tangle of these discrete forces, and this is the fabric.

The theory has shown success in explaining multiple physical phenomena across a range of particle, wave, and cosmological effects. However it is conjectural in nature and still in the process of development. Its current coverage includes wave-particle duality in the double slit device [9], derivation of optical laws from a particle perspective [9], explanation of the decay processes and prediction of a unified decay model [11] [12], explanation for the selective spin characteristics of neutrinos (whereby the direction of spin is correlated with the matter-antimatter species) [11], explanation for the annihilation process including the difference between otho- and para-positronium decay rates (ortho and para refer to spin combinations of the bound electron and anti-electron/positron) [13], provision of a mechanics for pair production [14] and likewise photon emission [15] [16], structure of atomic nuclei and explanation of stability for nuclides $\mathrm{H}$ to $\mathrm{Ne}$ [17] [18], prediction of a mechanism for asymmetrical baryogenesis in terms of a newly predicted decay path for remanufacture of the antielectron to the proton [19], proposed origin of entropy [20], a theory for time as an emergent property of matter rather than a universal attribute [21], nature of the vacuum and the cosmological horizon [22], and the origin of the finite speed of light [23]. The present paper extends the Cordus Theory by adding a quantitative derivation of the time and relativistic effects.

\section{Approach}

\subsection{Purpose}

The purpose of this work was to prospect for a relativistic formulation from the non-local hidden-variable theory provided by the Cordus Theory. This is worth attempting for the potential to unify aspects of particle behaviour and relativity. Furthermore, since the Cordus Theory is based on physical realism, a successful derivation might provide an explanation of relativistic motion and time dilation grounded in physical realism, hence elucidate deeper physics.

\subsection{Approach}

Prior work established that under this theory the vacuum speed of light [24] and 
the rate of time [21] are inversely related to fabric density which in turn is an emergent property of the spatial distribution of matter, hence varies with location in space. We extend this concept to derive the Lorentz transformation. We show that a specific property of the Cordus sub-particle structure, namely the flux tube of discrete force emissions, allows a novel and direct way to achieve this. The theory also predicts that the Lorentz formulation is modified by the fabric density. This requires the conventional concept of an inertial frame of reference to be extended to include the effect of fabric density. We then determine the implications for time dilation for relative motion, by building on prior qualitative work [21] which we extend to a quantitative formulation. The final stage is to derive the relativistic Doppler relationship for this particle theory. This is important because the transverse Doppler Effect is a unique prediction of relativity. We show that this prediction is also achievable from a particle perspective.

\section{Results}

\subsection{Derivation of the Lorentz Transformation}

\section{1) Assumptions}

Consider massy particule B (e.g. an electron) with a Cordus structure and travelling at constant velocity $v_{B}$ along the $\mathrm{x}$-axis, see Figure 2 . Let $\mathrm{B}$ emit discrete fields at a frequency, assume these propagate out radially at the local propagation speed, and assume this to be the speed of light $c$.

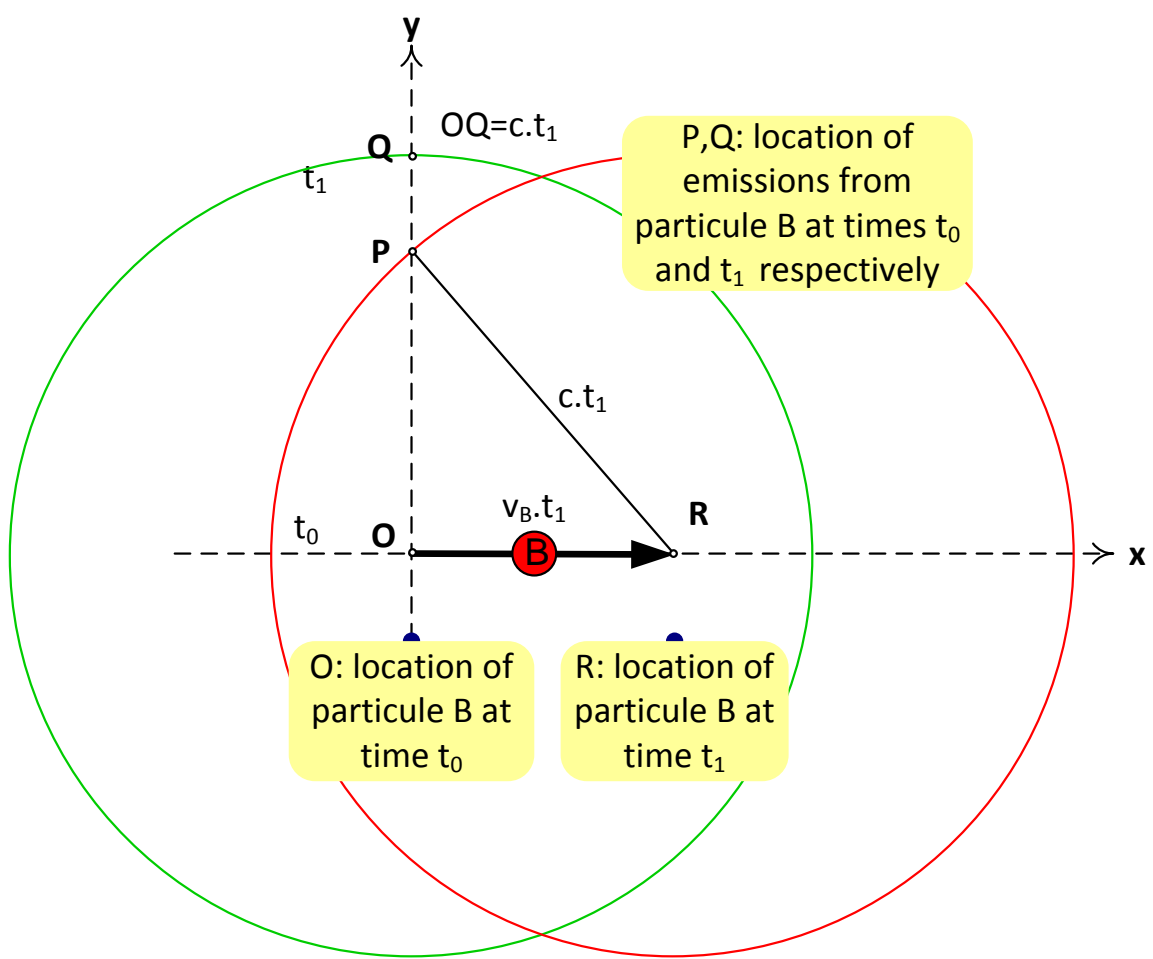

Figure 2. Geometric construction for Lorentz derivation. 


\section{2) Derivation of Lorentz from geometric considerations}

The derivation of the Lorentz transformation is achieved by geometric considerations of the effect of movement on the flux tube of discrete forces. Particule $\mathrm{B}$ passes point $\mathrm{O}$ at time $t_{0}$ and emits a discrete field at this moment. After time $t_{1}$ this field emission moves out radially on the $y$-axis a distance $c \cdot t_{1}$ to point $\mathrm{Q}$. In this same time $\mathrm{B}$ moves a distance $v_{B} \cdot t_{1}$ to point $\mathrm{R}$ on the $\mathrm{x}$-axis. $\mathrm{B}$ continues its field emission during this process. Were $\mathrm{B}$ to have been stationary at $\mathrm{R}$ instead, its emission would have reached point $\mathrm{P}$ in the same time. Note that the speed of propagation $c$ is finite.

The emission from $\mathrm{B}$ as it moves from $\mathrm{O}$ to $\mathrm{R}$ must have continuity of the flux tube rather than be broken. Hence the emission from location $\mathrm{R}$ is not absent at $\mathrm{Q}$, but is instead stretched, hence redshifted.

Then by geometric considerations of the distances between locations:

$$
\overline{\mathrm{RP}}^{2}=\overline{\mathrm{OR}}^{2}+\overline{\mathrm{OP}}^{2}
$$

Hence

$$
\overline{\mathrm{OP}}=\sqrt{\left(c t_{1}\right)^{2}-\left(v_{B} t_{1}\right)^{2}}=c t_{1} \sqrt{1-\left(\frac{v_{B}}{c}\right)^{2}}
$$

The extent of stretch is how far the emission from $\mathrm{R}$ has reached towards $\mathrm{Q}$, i.e. distance $\mathrm{OP}$, relative to where the emission from $\mathrm{O}$ has reached, i.e. distance OQ. Hence:

$$
\frac{\overline{\mathrm{OQ}}}{\overline{\mathrm{OP}}}=\frac{c t_{1}}{c t_{1} \sqrt{1-\left(\frac{v_{B}}{c}\right)^{2}}}=\frac{1}{\sqrt{1-\frac{v_{B}^{2}}{c^{2}}}}=\gamma
$$

This is the formulation for the Lorentz factor $\gamma$. This equation is consistent with the conventional expression of the Lorentz. This completes the first objective, which was to derive the Lorentz from a particule perspective. The derivation is based on plausible assumptions of the continuity of the flux tube.

\section{3) Physical interpretation}

As velocity increases closer to the speed of light, so $\gamma$ becomes larger. Greater $\gamma$ causes reduced apparent frequency of emissions of $B$ as perceived at $O, f_{B O b}$ compared to that at source $f_{B}$ :

$$
f_{B O b}=\frac{f_{B}}{\gamma}
$$

Although the derivation considered the stretch in the $\mathrm{y}$-axis, and assumed a time interval $t_{1}$, the size of the time interval is immaterial. In the limit as $\mathrm{Q}->\mathrm{O}$, i.e. for an Observer positioned at $\mathrm{O}$, the frequency change still exists. Note also that the situation from which the Lorentz is observed is the stationary point $\mathrm{O}$.

As $\mathrm{OP} \leq \mathrm{OQ}$ always, so $\mathrm{OQ} / \mathrm{OP} \geq 1$ and the stretch gets larger as the velocity $\mathrm{v}$ increases. Hence redshift increases with velocity.

The relativistic time dilation may be derived. In what follows the Observer Ob1 is positioned in situation 1 . The Lorentz, as derived above, is an indication 
of the stretch of the Cordus flux tube, and this stretch is manifest as slowing of the frequency as observed by Ob1, giving:

$$
f_{B O b 1}=\frac{f_{B 1}}{\gamma}
$$

where $f_{B 1}$ is the native frequency of object $\mathrm{B}$ in situation 1 . Frequency in the Cordus Theory also corresponds to the rate of time passing for a particule [21]. Greater $\gamma$ causes reduced frequency of emissions of $\mathrm{B}$ as perceived at $\mathrm{O}$, i.e. the clock of moving B appears to be slower to Ob1. This is consistent with the conventional formulation of special relativity whereby a stationary observer perceives a moving clock to run slower.

\section{4) Evaluation of assumptions}

We have assumed that $\mathrm{B}$ is a Cordus particule, and emits a discrete field at a frequency. This frequency emission is intrinsically included in the Cordus Theory. The theory proposes that particules emit discrete forces from one reactive end, and then from the other, with the emissions from a reactive end connected to make a flux tube. This construct for frequency is important because it provides the rationale for the emissions from $\mathrm{O}$ and $\mathrm{R}$ to be synchronized. This supports the interpretation of stretch of the flux tube. To achieve something similar within the conventional framework of general relativity, one could assume B to emit pulses of light. However it then becomes difficult to explain why such pulses of light should have temporal continuity between them.

While the Cordus Theory proposes that particule B has two reactive ends separated by a span, the above derivation takes the macroscopic perspective and assumes that the span is negligible. We have also assumed B to be a single particle rather than a macroscopic body of many particles. However this is only for convenience of explanation, because it means that only one field is emitted, rather than many. A macroscopic body simply emits many overlapping discrete fields. Each of these is individually subject to the same considerations given here. Thus the derivation applies to assemblies of multiple particles and macroscopic bodies whether coherent or decoherent.

Up to here it has been assumed that the speed of outward propagation of a field is the speed of light. This is consistent with conventional assumptions. However the Cordus Theory predicts that the speed of light is not universally constant but instead depends on the local fabric density [21]. In the next section we take this into account.

\subsection{Time and Fabric Density}

\section{1) Effect of fabric on propagation of light}

Fabric refers to the mesh of moving flux tubes that are postulated to exist in space [22]. These flux tubes comprise the discrete forces emitted by all particules in the accessible universe. In physical terms, this refers to the magnitude of the gross (not the net) electric field in space, under the assumption that even neutral charges emit positive and negative fields. The fabric also distinguishes the va- 
cuum within the universe from the void before genesis, and is proposed as the reason for the existence of electrical and magnetic constants of the vacuum. The density of the fabric $\phi$ determines those constants and gives the local speed of light a specific value [23].

At a physical level, the theory proposes that the speed of light arises as the ability of the photon to advance through the fabric. This act of locomotion is proposed to involve the evanescent field of the photon recruiting the fabric's discrete forces, hence creating a travelling disturbance in the fabric. The greater the fabric density the more discrete forces to disturb, and hence in a unit time the photon makes shorter incremental displacement along its locus, hence the slower light travels. ${ }^{1}$ With this fabric $c$ is locally invariant for isotropic $\phi$, hence the speed of light is the same regardless of (1) the motion of the observer or light source, or (2) the direction of motion. However it also follows that under these assumptions the fabric density must generally be variable, since it depends on the local distribution of mass, which is not homogenous. Consequently the theory predicts that $\phi$ varies with location in space. This also means that the speed of light is not strictly constant but is instead dependent on the spatial distribution of matter [23].

For this theory, the single-body gravitational field approximates to the fabric density. In the more complex case of $n$-bodies then the mutual contributions increase $\phi$ but weaken the net gravitational field, so the correspondence is broken. Nonetheless the simple single-body case is useful for exploring the phenomena.

The concept of fabric is comparable to the virtual particles of the vacuum in quantum mechanics, though the concepts are not used the same way. Likewise, others have suggested that the distribution of matter affects general relativity [25].

\section{2) Theory of time}

The Cordus Theory proposes that time arises due to the frequency emissions of particles [21]. This may need some explanation as it is a different concept to that of general relativity where time is a dimension. The Cordus Theory posits that time is the rate at which a particule is able to energize and emit discrete forces. These phases of energization are also when the particule responds to the discrete forces of other particules. This is the only way the particule can interact with its surroundings. The particule only exhibits agency-the ability to interact with another particule or field-when it is energized.

Particules that are able to energize faster will achieve more of these interactions than identical particules elsewhere that energize at a slower frequency: the latter are time dilated. The phenomenon of time therefore arises at the sub-particule level, and via these interactions scales up to the macroscopic level. Thus, for example, the mechanical process of the ticking of a clock is linked to ${ }^{1}$ In contrast, a higher density of the medium results in faster speed for sound. However these have different underlying mechanisms. For light the disturbance in the fabric requires the recruitment of a lateral volume of medium, whereas for sound the disturbance involves compression-tension of the medium in the axial direction. 
the rate at which its atoms are able to respond to internal and contact forces, and these depend on the fundamental interactions. This means that the rate of time must be local to the situation: the theory precludes the existence of a universal time across the universe, nor does it accept that time is a dimension.

The frequency of the particule, hence its time rate, is affected by the ease with which the particule can emit its discrete fields into the fabric. This is affected by multiple factors. First is the background fabric density for that situation in space, which includes but is more than the gravitational field. Second is the relativistic velocity whereby forward emitted discrete forces locally densify the fabric. Third is that any acceleration of the particule increases its rate of engagement with the fabric. The total fabric density relative to the particule is the sum of these. Thus increased fabric density may arise from either a more matter-rich region of space, or higher velocity, or acceleration, or a stronger gravitational field. These are predicted to cause the particule's own emissions of discrete forces to be retarded, hence its frequency to be slower. Thus also the rate of time is slower for the particule. The theory thus gives a qualitative explanation for the causality of time dilation at the particle level [21].

\section{3) Definition of situation}

The fabric density $\phi$ is therefore a new variable to be included in the Lorentz formulation. This variable is not expressed in orthodox cosmology, which assumes that the vacuum properties are universally and temporally isotropic. The Cordus Theory rejects this idea of cosmological homogeneity, and proposes instead that there is a gradient of fabric density across the universe due to the historic expansion thereof [22], and due to the non-homogenous spatial distribution of matter.

Whereas relativity assumes that two inertial frames of reference are equivalent, the Cordus Theory instead proposes that equivalence only applies if the fabric densities are also the same. This cannot generally be accepted to be the case, at least not at cosmological scales or involving different epochs. Consequently we introduce the term "situation" to describe an inertial frame of reference with a specific fabric density. Two situations are only similar if both their inertial kinematics and fabric densities are the same.

Note that in the derivations so far the fabric density $\phi$ was assumed to be constant throughout. Thus particule B started and continued in the same fabric density, which was also the same at all points under consideration. In the more general case the particule B starts in situation 1 and subsequently moves into situation 2 of different fabric density. We derive this formulation next.

\section{4) Lorentz fabric density transformations}

The Cordus Theory predicts that the frequency of a massy particule is inversely related to fabric density. We propose as a lemma that the relationship is one of inverse proportionality rather than any other function:

$$
f \sim \frac{1}{\phi}
$$


This leads to the following relationships of situational relativity.

\section{a) Intrinsic changes as the observer moves into a different fabric density}

In the general case consider massy particule $B$ with non-relativistic velocity $\mathrm{v}_{\mathrm{B} 1}$ starting in situation 1 with fabric density $\phi_{1}$ and frequency $f_{B 1}$. It subsequently moves into situation 2 of different fabric density $\phi_{2}$ where its velocity becomes $V_{B 2}$ and its frequency $f_{B 2}$ as measured by a co-moving observer Ob2 in situation 2 . These are termed intrinsic changes because the properties of $\mathrm{B}$ change, even though the observer travelling with $B$ does not notice them. In applying the intrinsic transformations, it is assumed that Object B was once in one situation and then moved to another.

\section{b) Frequency of massy particule}

The frequency of massy body B as measured in the new situation (i.e. the point of observation is co-moving with $\mathrm{B}$ ) changes to:

$$
f_{B 2} \phi_{2}=f_{B 1} \phi_{1}
$$

This is because frequency at the fundamental level is the rate at which time passes for the particule, according to this theory. If the Observer travelling with particule B moves into a situation $\phi_{2}$ of lower fabric density, then the fabric resistance to the emission of discrete forces reduces, so the emission frequency increases. Consequently all processes are faster in situation 2, i.e. the time rate is faster. The particule has greater agency to interact with other particules. This also means that an observer $\mathrm{Ob} 2$ in situation 2 of lower fabric density is able to process information faster than an observer Ob1 in situation 1. Consequently $\mathrm{Ob} 2$ does not perceive self to be operating at faster time, but rather that $\mathrm{Ob} 1$ and objects in situation 1 have relatively slower rate of time. This applies to massy particules, including clocks, that travel with the Observer into situations of different $\phi$

\section{c) Velocity of massy particule}

The velocity of $B$ as measured in situation 2 becomes:

$$
v_{B 1} \phi_{1}=v_{B 2} \phi_{2}
$$

Thus we are proposing that there is an intrinsic change in both frequency and velocity, i.e. that the particule really does change those properties. However to the particule itself, the change in its own frequency is not apparent. This is because its frequency also determines its rate of time. For example, velocity $v_{B}$ increases when B moves into a situation of lower fabric density, but its frequency, and hence rate of time, also increases by the same proportion. So the co-moving distance travelled by B in a unit of own time is the same as before, though both the velocity and the unit of time have changed. As the intrinsic change in velocity is not perceived by co-moving Ob2, consequently there is predicted to be no perception of inertial acceleration. It is only by examining own progress relative to background objects in situation 1 that $\mathrm{Ob} 2$ can infer own velocity to have increased. Alternatively, Ob2 perceives objects in situation 1 to have length contraction. 


\section{d) Behaviour of light in situations of changing fabric density}

So far the focus has been on the behaviour of massy particules. The photon is predicted to behave differently, because the speed of light $c$ is the saturated speed of propagation of discrete forces. The Cordus Theory predicts that $c$ is inversely related to the fabric density [24]. The theory does not predict the form of this relationship, so we proceed on the assumption of a simple inverse proportionality with fabric density. Hence the speed of light as it moves from situation 1 to 2 is:

$$
c_{2} \phi_{2}=c_{1} \phi_{1}
$$

For example, if $B$ moves into a situation $\phi_{2}$ of lower fabric density, then the local speed of light $c_{2}$ increases, though it remains relativistic (is not affected by the velocity of the emitting particule) and is homogenous within that situation providing there is no gradient to the fabric density. Consequently the Cordus Theory is a variable speed of light (VSL) theory [23]. There are other VSL theories in physics, but the Cordus Theory is unique in predicting that the variability originates with fabric density hence matter distribution.

5) Applications

a) Apparent changes due to observation from a situation of different fabric density

The other transformation is when an object B in situation with $\phi_{2}$ is remotely viewed by an Observer Ob1 who remains in situation 1 with fabric density $\phi_{1}$. Ob1 may have no knowledge of the past history of $\mathrm{B}$, or when in the past they inhabited a common situation, i.e. when their temporal-spatial trajectories last converged.

Observer Ob1 observes the passage of B across a foreground of situation 1 marker objects of spacing $L$, and then uses the local time, i.e. frequency $f_{1}$, in situation 1 to infer the velocity of $\mathrm{B}$ :

$$
v_{B O b 1}=L f_{1}
$$

The fabric density effects are not dependent on velocity in the non-relativistic case, so the effect described here is not the same as Lorentz-Fitzgerald length contraction which is purely a velocity effect. Object B notes its own passage against the same marker objects, the spacing of which is also L. For explanation assume $\phi_{2}<\phi_{1}$ hence frequency $f_{2}$ is faster in situation 2 (per Equation (2.2)). Thus B assesses its own velocity $V_{B 2}$ as the distance travelled per (shorter) unit of time:

$$
v_{B 2}=L f_{2}
$$

Consequently both the frequency $f_{2}$ (and hence rate of time passing) and velocity $V_{B 2}$ are increased for Object $B$ when it is in a situation of lower fabric density $\left(\phi_{2}<\phi_{1}\right)$. Thus the velocity of $B$ is higher than $V_{B O b 1}$ by the ratio of fabric densities. We have thus:

$$
\frac{v_{B O b 1}}{f_{1}}=\frac{v_{B 2}}{f_{2}}
$$


Hence also:

$$
v_{B O b 1} \phi_{1}=v_{B 2} \phi_{2}
$$

This is the same general form as Equation (2.3). This is a useful equation as it allows the velocity $v_{B 2}$ of $\mathrm{B}$ to be determined from its apparent value in another situation, providing the ratio of fabric densities is known. If there are no Doppler or relativistic velocities then the ratio may be determined by the observed frequencies of some characteristic electron/photon effect. If a time rate $f_{B 2}$ is observed from situation 1, then Equation (2.2) applies.

\section{b) Round trip}

The transformations may be applied sequentially. Consider identical Objects A and B that initially share with Observer Ob1 a common situation 1 with $\phi_{1}$. B has initial velocity $\mathrm{v}_{\mathrm{B} 1}$ and frequency $f_{B 1}$, and the properties of $\mathrm{A}$ are initially the same as those of $B$. Object A remains moving in situation 1 , but B moves into situation 2 with fabric density $\phi_{2}$. Assume for explanation that $\phi_{2}<\phi_{1}$. Then the velocity of $\mathrm{B}$ increases to $v_{B 2}=v_{B 1} \phi_{1} / \phi_{2}$ per Equation (2.3). Observer Ob1 remains in situation 1 and observes the passage of $B$ against a foreground of situation 1 markers, and measures velocity $\mathrm{v}_{\mathrm{BOb} 1}$ using Ob1's (slower) rate of time.

Then the proper velocity of $\mathrm{B}$ is the distance as measured by $\mathrm{Ob} 1$ divided by the elapsed time as recorded by $B$. This may be inferred as $v_{B O b 1}=v_{B 2} \phi_{2} / \phi_{1}$ per Equation (2.8). Substitute $v_{B 2}$ from Equation (2.3) hence $v_{B O b 1}=v_{B 1}$. Thus the proper velocity of $B$ is the same as before. The proposed physical explanation is that the decrease in fabric density for B causes the intrinsic velocity of B to increase, but also increases the clock frequency of B by the same proportion. This is the combined effect of B moving out of situation-1 and then being observed from situation 1 . Thus B will perceive that its speed is unchanged, whereas Ob1 will perceive $B$ to be moving faster in the lower fabric density situation.

If $B$ subsequently returns to situation 1, its velocity will decrease per Equation (2.3) as it enters the higher fabric density $\phi_{1}$, and the observational difference will collapse per Equation (2.5), so it will once more take up velocity $v_{B 1}$ and in that respect be the same as Object $A$. There will no longer be any difference in simultaneity between A and B. However B will have accumulated more frequency cycles than A, per Equation (2.2), and hence B will have aged more than A.

This explanation has been given in terms of fabric density. The general principles are proposed to apply also to gravitational fields, which are a proxy for $\phi$. Thus if $\mathrm{B}$ moves with non-relativistic motion into a region of lower gravity, then conventional gravitational time dilation expects that $\mathrm{B}$ will experience faster time frequency. This is consistent with the Cordus explanation provided above. However the Cordus Theory also predicts that B will move faster too, which is a new prediction. This has implications for the equivalence of gravitational and inertial mass.

\section{c) Gravitational time dilation for massy particules}

Consider a massy particule $B$ that moves away from a massive object, hence 
moving from a stronger gravitational field (situation 1) to a weaker field (situation 2). For the simple case of a universe with a lumped central mass, the gravitational field corresponds exactly to the fabric density. Hence this distal movement corresponds to a decrease from $\phi_{1}$ to $\phi_{2}$ and Equation (2.2) predicts a corresponding increase in the intrinsic frequency of the particule. This results in a faster rate of time for the particule in situation 2 compared to one in a stronger field. Since a macroscopic body B comprises many fundamental particules this means that the body ages faster in lower gravitational field. This is consistent with gravitational time dilation per general relativity where a frame must be moving with the escape velocity $v_{\text {esc }}$ and per the Schwarzschild metric this is:

$$
v_{\text {esc }}=\sqrt{\frac{2 G M}{r}}
$$

with $G$ gravitational constant, $M$ mass of the central body, $r$ radial position of the Observer's location from the centre of the body. Hence per Equation (1.3)

$$
\gamma=\frac{1}{\sqrt{1-\frac{2 G M}{r c^{2}}}}
$$

which is the Lorentz gravitational transformation. However the present theory requires that neither $G$ nor $c$ be universal constants, hence the Schwarzschild formulation of gravitational time dilation is expected to be a simplification of a more complex formulation yet to be elucidated.

\section{d) Gravitational redshift of photons}

The previous case was for a massy particule experiencing a changing gravitational field: the frequency of the particule increases as it moves outward and this is evident in its changing rate of time. Different behaviour arises where the outward-moving particule is a photon, and in this case redshift occurs, for the following reasons.

When a photon moves outwards against a gravitational field, it moves from situation 1 with higher $\phi_{1}$, into a situation 2 of lower $\phi_{2}$ where Observer Ob2 is located. The conventional prediction is that the photon's frequency will reduce as it moves outwards, hence it will be red shifted. Our Equation (2.2) appears to be contrary to this, as it predicts that the frequency will increase as viewed in the co-moving frame, but that equation only applies to massy particules. For a photon, the lower fabric density causes an increase in the velocity per Equation (2.4), hence a stretch of the wavelength and a reduction in frequency. Thus the Cordus Theory also predicts that the photon will display gravitational redshift, though attributes this to the change in fabric density rather than the gravitational field per se. Thus the Cordus Theory makes the falsifiable prediction that the gravitational red-shift will depend not only on the gravitational potential but also on the background fabric density. For situations with higher background $\phi$ the extent of the redshift will be reduced. 


\subsection{Relativistic Velocity with Changing Fabric Density}

Previous sections have separately derived the Lorentz velocity transformation and the fabric density transformations. Now these are combined.

\section{1) Geometric considerations}

Consider the arrangement per Figure 2, but now assume point $\mathrm{R}$ is in a situation of fabric density $\phi_{2}$, whereas the observer $\mathrm{Ob} 1$ at $\mathrm{O}$ is in a situation with fabric density $\phi_{1}$. Assume that the fabric density changes abruptly immediately outside point $\mathrm{O}$. Then the field emission from object $\mathrm{B}$ travelling along RP will be subject to $V_{B 2}$ and $c_{2}$. Hence the propagation distance OP becomes:

$$
\overline{\mathrm{OP}}=\sqrt{\left(c_{2} t_{1}\right)^{2}-\left(v_{B 2} t_{1}\right)^{2}}
$$

Then substitute the fabric density transformations to refer $c_{2}$ to $c_{1}$ (Equation (2.4)) and $v_{B 2}$ to $v_{B 1}$ (Equation (2.2)):

$$
\overline{\mathrm{OP}}=\sqrt{\left(c_{1} \frac{\phi_{1}}{\phi_{2}} t_{1}\right)^{2}-\left(v_{B 1} \frac{\phi_{1}}{\phi_{2}} t_{1}\right)^{2}}=c_{1} \frac{\phi_{1}}{\phi_{2}} t_{1} \sqrt{1-\frac{v_{B 1}^{2}}{c_{1}^{2}}}
$$

The speed of light at $\mathrm{O}$ determines the distance OQ:

$$
\overline{\mathrm{OQ}}=c_{1} t_{1}
$$

So the Lorentz for variable fabric density, as perceived by $\mathrm{Ob} 1$ at point $\mathrm{O}$, is:

$$
\frac{\overline{\mathrm{OQ}}}{\overline{\mathrm{OP}}}=\gamma(\phi)=\frac{1}{\left(\frac{\phi_{1}}{\phi_{2}}\right) \sqrt{1-\frac{v_{B 1}^{2}}{c_{1}^{2}}}}
$$

Thus this particle mechanics requires the relativistic Lorentz to have an additional factor included, which is the ratio of fabric density between the two situations. Thus as velocity increases closer to the speed of light, so $\gamma(\phi)$ becomes larger, as per the usual Lorenz effect. However, as the fabric density in the situation 2 decreases, so $\gamma(\phi)$ becomes smaller. The Lorentz $\gamma(\phi)$ is maximized by higher velocity and movement into situations of higher fabric density. In both cases the moving particule experiences the fabric at a greater rate. The implication is that the conventional Lorentz is an incomplete representation of relativistic phenomena, since it only includes the velocity component and omits the situational differences caused by fabric density. In the case where there is no difference in fabric density, then the conventional Lorenz is recovered. The novel prediction here is that the underlying causality for relativistic effects includes not only velocity, but also the fabric density. This is not anticipated by other theories, and is a falsifiable prediction of the Cordus Theory.

\subsection{Time Dilation with Relativistic Velocity and Variable Fabric Density}

Previous work on the Cordus Theory for time dilation anticipated the effect of fabric density, but the treatment was primarily conceptually [21]. Now we develop a quantitative formalism. Consider an Observer Ob1 (in situation 1 with 
fabric density $\phi_{1}$ ) who observes body B (in situation 2 with fabric density $\phi_{2}$ and moving with velocity $V_{B 2}$ measured in 2 , or $V_{B O b 1}$ measured in 1). Then the frequency (rate of time) of $\mathrm{B}$ as perceived remotely by $\mathrm{Ob}_{1}$ is by substitution of Equation (3.4) into Equation (1.4):

$$
f_{B O b 1}=f_{B 2} \frac{1}{\gamma(\phi)}=f_{B 2}\left(\frac{\phi_{1}}{\phi_{2}}\right) \sqrt{1-\frac{v_{B 1}^{2}}{c_{1}^{2}}}
$$

This equation provides the mathematical formalism for the Cordus time-dilation concept.

The implications are that time dilation is determined by both velocity and fabric density. Note that fabric density is determined by the spatial distribution of matter in the accessible universe around the location under examination. Thus the fabric density is proposed to be the deeper causal mechanism that subsumes gravitational time dilation.

A remote clock B ticking at frequency $f_{B 2}$ and moving with velocity $V_{B 2}$ in situation 2 will be perceived by a remote observer to have a frequency $f_{B O b 1}$ that is determined not only by the Lorentz velocity factor, but also by the ratio of fabric density. The only variable that is unapparent from the perspective of Observer Ob1 is the remote fabric density $\phi_{2}$. This is a covert variable.

Hence we make the novel and falsifiable prediction that there is a time dilation due to a change in fabric density alone, even for particles at rest. The formulation is obtained by putting $V_{B 1}=0$ in Equation (4.1):

$$
f_{B O b 1}=\frac{f_{B 2}}{\gamma}=f_{B 2}\left(\frac{\phi_{1}}{\phi_{2}}\right)
$$

for $v_{B 1} \ll c_{1}$

This may also be recovered from combining Equations (2.7) and (2.8). In the case where $\phi_{2}$ is less than $\phi_{1}$, then observer Ob1 will perceive B to have a greater frequency than if B was in the same situation as Ob1.

In these equations $f$ refers to the energization frequency of the single particule under consideration. A macroscopic body has many particules that energize at different frequencies, which all scale by the same factor $\gamma(\phi)$. The Cordus Theory identifies that particules are only reactive when they energize, i.e. that the agency of a particule occurs at its frequency. Hence all interactions between particules in the body are scaled identically. These interactions include strain, kinematics, chemical reactions, field forces, and nuclear processes. Consequently the body as a whole experiences a change in its rate of time, and this applies whether or not the body has life or its state of coherence. Thus the time interval $\Delta t$ between two ticks of a clock depends on its internal interactions and these are moderated by $\gamma(\phi)$, regardless of whether the mechanism is mechanical, electrical or atomic.

Consider stationary body A in situation 1, and moving body B in situation 2 . Both are equipped with identical clocks that according to $\mathrm{A}$ beat with intervals of $\Delta t_{1} \propto 1 / f_{A 1}$. Since the clocks are identical A believes that $f_{B 2}=f_{B 1}=f_{A 1}$. Then 
A will observe that the clock of $B$ is time dilated to interval $\Delta t^{\prime}$ as follows. Per Equation (4.1) put $\Delta t^{\prime}=1 / f_{B O b 1}$ and $\Delta t_{1}=1 / f_{B 2}$ hence we have:

$$
\Delta t^{\prime}=\Delta t_{1} \frac{1}{\left(\frac{\phi_{1}}{\phi_{2}}\right) \sqrt{1-\frac{v_{B 1}^{2}}{c_{1}^{2}}}}
$$

Thus the moving clock B will be perceived to have a longer interval $\Delta t^{\prime}$ if it moves faster (higher $v_{B 1}$ ) or moves into a region of greater fabric density (greater $\phi_{2}$ ). Note that $v_{B 1}$ is the proper motion of B in situation 2, from the perspective of an observer in situation 1 . This recovers the conventional kinematic time-dilation formula, but with the addition of a fabric effect.

\subsection{Doppler Change in Perceived Frequency}

The above formulations show how frequency is affected by relativistic velocity and fabric density together. However there is also the Doppler Effect to include, since this also changes frequency. We start by showing how the conventional Doppler equation may be derived from the Cordus particule basis, and then progress to add the fabric density effect.

\section{1) Derivation of conventional Doppler Effect (no fabric gradient)}

Consider all actions occurring in situation 2. Body B moves with constant velocity $\mathrm{v}_{\mathrm{B} 2}$, and the frequency of its light is detected by an observer Ob2 from within the same situation (inertial frame of reference with constant fabric density). This motion results in a Doppler shift of the emitted frequency. Assume that the motion of $\mathrm{B}$ does not take it out of situation 2, i.e. B continues to experience the same background fabric density $\phi_{2}$. The velocity $V_{B 2}$ may be resolved into a component in the line of sight of and towards the observer $V_{B 2 / /}$, and a component transverse to the line of sight $V_{B 2 T}$.

\section{2) Descriptive explanation}

The change in frequency (e.g. increases for bodies with closing velocity) is a result of the Observer encountering more wavelength fronts in a given time. The wave source (or Observer) has time to move before the next wave is intercepted. If waves travelled instantly then the movement to a new emitting (or observing) location would make no difference to the receipt of the next wave since it would arrive instantly after it was emitted.

Then the Doppler shifted wavelength of light from B as perceived by Ob2, which is $\lambda_{B O b 2}$, is the native wavelength $\lambda_{B 2}$ less that reduced by the ratio of the parallel velocity $V_{B 2 / /}$ to the local speed of light $c_{2}$ :

$$
\lambda_{\mathrm{BOb2}}=\lambda_{\mathrm{B} 2}-\frac{v_{\mathrm{B}_{2}}}{c_{2}} \lambda_{\mathrm{B} 2}=\lambda_{\mathrm{B} 2}\left(1-\frac{v_{B_{2}} / /}{C_{2}}\right)
$$

Hence the apparent Doppler frequency of $\mathrm{B}$ as perceived by $\mathrm{Ob} 2$ is:

$$
f_{B O b 2}=\frac{c_{2}}{\lambda_{B O b 2}}=\frac{c_{2}}{\lambda_{B 2}\left(1-\frac{v_{B_{2} / /}}{c_{2}}\right)}=\frac{f_{B 2}}{\left(1-\frac{v_{B_{2} / l}}{c_{2}}\right)}
$$


This derives the conventional Doppler equation. The Doppler factor may be written as the ratio of the observed frequency $f_{B O b 2}$ to the emitted frequency at source $f_{B 2}$.

\subsection{Relativistic Doppler with Fabric Density}

The next objective is to combine all three effects: Lorentz, Doppler, and fabric density. The relativistic Doppler can be handled very well in general relativity. It is a key prediction and success of relativity, and a point of difference with Newtonian mechanics. However it cannot be handled so well from the particle perspectives of QM or string theory. Nor has this previously been achieved using NLHV theory.

\section{1) Doppler with time dilation}

Consider object B moving with velocity $V_{B 2}$ in situation 2 of fabric density $\phi_{2}$ and local speed of light $c_{2}$. Object B emits light at a native frequency $f_{B 2}$ or wavelength $\lambda_{B 2}$, these being as measured by $\mathrm{B}$ itself. This emission is received by remote Observer Ob1 located in situation 1 having fabric density $\phi_{1}$ and local speed of light $c_{1}$.

a) Approach

Construct the analysis by progressively changing the vantage point of the Observer, from $\mathrm{Ob} 2$ with the object in situation 2, to Ob1 in situation 1. Apply the relevant transformations at each stage. Also introduce the concept of expected frequency.

\section{b) Analysis of situation 2}

Start with Doppler (Equation (5.2)), and consider an Observer Ob2 in situation 2. Note that $f_{B 2}$ is the emitted frequency at source. In this case, where there is no fabric gradient within the same situation, $\mathrm{Ob} 2$ has perfect knowledge about the expected value of frequency $f_{B 2}$ because this can be measured for an equivalent stationary object, providing the emission phenomenon can be replicated. Hence $f_{B 2}$ equals the value $f_{B E x p 2}$ expected for the phenomenon by an Observer Ob2 in situation 2. In other words, the Observer has additional information about the 'true' nature of the emission at source. This assumption of universality is tacitly built into the conventional Doppler equation. We propose that no such truth is knowable unless the fabric densities are also known.

Note also that the velocity $\mathrm{v}_{\mathrm{B} 2}$ is as $\mathrm{B}$ perceives its own motion, e.g. against a backdrop of stationary marker objects in situation 2 . This is also the velocity observed by co-moving Ob2, since there is no time dilation within this situation. Thus $v_{B 2}=v_{B O b 2}$. The Doppler Equation (5.2) becomes:

$$
f_{B O b 2}=f_{B E x p 2} \frac{1}{\left(1-\frac{v_{B O b 2 / /}}{c_{2}}\right)}
$$

\section{2) Equivalent values in situation 1}

Now change the perspective and consider an Observer Ob1 in situation 1, who is looking at object B in situation 2. It is necessary to apply the Lorentz time di- 
lation with fabric density, per Equation (4.1). Note that $f_{B 2}=f_{B O b 2}$ for reasons above, and thus substitute Equation (6.1) into Equation (4.1). Hence:

$$
f_{B O b 1}=f_{B E x p 2} \frac{1}{\left(1-\frac{v_{B O b 2 / /}}{c_{2}}\right)}\left(\frac{\phi_{1}}{\phi_{2}}\right) \sqrt{1-\frac{v_{B 1}^{2}}{c_{1}^{2}}}
$$

This is incomplete as it is still necessary to refer all situation 2 parameters back to the equivalents in situation 1 using the fabric density transformations (Equation (2)).

\section{a) Expected frequency $f_{B \operatorname{Exp} 2}$}

A crucial component is the expected frequency and its dependency on fabric density. This frequency is based on a phenomenon, e.g. an electron energy change with a characteristic photon emission. The conventional assumption is that this frequency is universally the same at all sources, and hence that any observed differences may be attributed only to Doppler or relativistic effects hence to velocity difference. However the Cordus Theory rejects that interpretation as simplistic, and instead predicts that frequency and the rate of time are dependent not only on velocity but also on background fabric density. For stationary Object B in situation with lower $\phi_{2}$ the frequency of all interactions is higher, and thus the same physical emission phenomenon also occurs at a higher frequency. Hence $f_{B E x p 2}$ in situation 2 is higher than the frequency $f_{A E x p 1}$ of the same phenomenon in situation 1 . The expected frequency of the phenomenon in situation 1 is therefore affected by the fabric density per intrinsic changes Equation (2.2). Hence:

$$
f_{\text {BExp } 2}=f_{A \operatorname{Exp} 1} \frac{\phi_{1}}{\phi_{2}}
$$

This takes care of the static situation where there is a fabric difference between the situations, but no motion. The relativistic velocity effects are accommodated in the Lorentz.

\section{b) Doppler velocity $\mathbf{v}_{\mathrm{B} 2 / /}$}

The observer Ob1 in $\phi_{1}$ measures velocity $v_{B 1 / /}$ rather than the native $v_{B 2 / /}$ itself, and there are different way to reconcile this. We suggest the following. For the Doppler component, the shift is related to the velocity of B relative to the speed of light in the same situation 2, per Equation (5.1). When deriving the Lorentz with fabric density at Equation (3.2) we used the fabric density transformations to refer $c_{2}$ to $c_{1}$ (Equation (2.4)) and $V_{B 2}$ to $V_{B 1}$ (Equation (2.3)), thereby determining the equivalent velocities in situation 1 . The same approach is applied here.

The ratio of fabric densities affects both $v$ and $c$ in the same way: both will be elevated by the same proportion if situation 2 has lower fabric density. Thus the fabric effect cancels. Thus we directly transfer the Doppler ratio for situation 2 into situation 1 : 
$\frac{v_{B O b 2 / /}}{c_{2}}=\frac{v_{B O b 1 / /}}{c_{1}}$

\section{c) Velocity in situation 1}

For the Lorentz component the observed speed is the same as used in Equation (3.2) hence:

$$
v_{B O b 1}=v_{B 1}
$$

\section{3) Resulting formulation of observed frequency}

To determine the observed frequency at Ob1, substitute Equation (6.3)-(6.5) into Equation (6.2):

$$
f_{B O b 1}=f_{A E x p 1} \frac{1}{\left(1-\frac{v_{B O b 1 / /}}{c_{1}}\right)}\left(\frac{\phi_{1}}{\phi_{2}}\right)^{2} \sqrt{1-\frac{v_{B O b 1}^{2}}{c_{1}^{2}}}
$$

This is the relativistic Doppler with fabric density.

Note that this formulation predicts a squared dependency on the ratio of fabric density, which is original. In other respects the conventional relativistic Doppler is recovered when there is no gradient in fabric density $\left(\phi_{1}=\phi_{2}\right)$.

This also recovers the transverse relativistic Doppler, i.e. there is a Doppler effect even when there is no component of motion in the line of sight $\left(v_{B O b 1 / /}=0\right)$. Equation (6.6) gives the transverse redshift as observed by Ob1 watching object B. The light emitted by B in its own situation is blue shifted because $\mathrm{B}$ emitted the light before the point of closest approach (point $\mathrm{O}$ in Figure 2 ), and hence the flux tube is compressed rather than stretched, per Equation (1.3).

This equation accomplishes the purpose of this paper. It derives the Lorentz transformation, including the fabric density. This provides a formalism for relativistic velocity time dilation.

4) Special case: motion in the line of sight

In the special case where the velocity of B is entirely in the line of sight, then $v_{B O b 1 / /}=v_{B O b 1}$, hence Equation (6.6) becomes:

$$
f_{B O b 1}=f_{A E x p 1} \frac{1}{\sqrt{\left(1-\frac{v_{B O b 1}}{c_{1}}\right)^{2}}}\left(\frac{\phi_{1}}{\phi_{2}}\right)^{2} \sqrt{1-\frac{v_{B O b 1}^{2}}{c_{1}^{2}}}=f_{A E x p 1}\left(\frac{\phi_{1}}{\phi_{2}}\right)^{2} \sqrt{\frac{1+\frac{v_{B O b 1}}{c_{1}}}{1-\frac{v_{B O b 1}}{c_{1}}}}
$$

This also has implications for the red-shift, as shown next.

\section{5) Redshift}

The conventional representation of the redshift may be recovered, by introducing simplified terminology to recast the equations into the conventional variables. Define:

$$
\begin{gathered}
f_{o}=f_{\text {BOb1 }} \\
f_{\text {Exp }}=f_{\text {AExp } 1}
\end{gathered}
$$




$$
v=v_{B O b 1}
$$

defined as positive toward $\mathrm{Ob} 1$.

$$
\begin{gathered}
c=c_{1} \\
\beta=v / c
\end{gathered}
$$

If $v$ is instead defined as positive away from Ob1 then the sign of $\beta$ reverses. Then Equation (6.7) becomes:

$$
f_{O}=f_{\operatorname{Exp}}\left(\frac{\phi_{1}}{\phi_{2}}\right)^{2} \sqrt{\frac{1+\frac{v}{c}}{1-\frac{v}{c}}}
$$

Hence the red-shift $(z)$ for the special case of motion in the line of sight is:

$$
z(\phi)=\frac{f_{\text {Exp }}-f_{O}}{f_{O}}=\frac{f_{\text {Exp }}}{f_{O}}-1=\left[\left(\frac{\phi_{2}}{\phi_{1}}\right)^{2} \sqrt{\frac{1-\frac{v}{C}}{1+\frac{v}{c}}}\right]-1=\left[\left(\frac{\phi_{2}}{\phi_{1}}\right)^{2} \sqrt{\frac{1-\beta}{1+\beta}}\right]-1
$$

Thus the red-shift is proposed to also depend on the fabric density ratio.

As this shows, it is possible to derive the Lorentz transformations from the particle perspective of the Cordus Theory.

\section{Discussion}

\subsection{Findings}

We propose on theoretical grounds that the conventional formulation of the Lorentz transformation is incomplete and needs the inclusion of a fabric density variable. General relativity is premised on the speed of light in-vacuuo being universally constant. In turn that premise arose as a simple way to formulate the relativity of simultaneity, and as a consequence of rejection of the aether following the Michelson-Morley experiment [26]. In contrast our theory splits and treats separately the relativity of observation, versus the speed of light. It proposes that the fabric is relativistic in terms of exhibiting Lorentz effects, and that the speed of light is constant within an isotropic fabric, but proposes that the fabric density is fundamentally anisotropic and that this affects the speed of light.

It is radical to propose fabric density as a new cosmological variable, but it is logically congruent with gravitational time-dilation. Gravitational field strength is then a proxy variable for fabric density. In principle this means that fabric density becomes the common cause of relativistic velocity effects and gravitational effects, hence may provide a means to explain the correspondence of inertial and gravitational mass.

The further implication is that general relativity is only applicable where the reference frames have the same fabric density, i.e. the situations are the same. This means that general relativity is expected to be accurate within the environs of the solar system, but not to intergalactic space. This has further implications 
for interpreting gravitational interactions at the galactic scale and larger.

We propose that it is necessary to abandon the cosmological principle with its assumption of homogeneity across the temporal phases and spatial dimensions of universe. In its place we propose the concept of variable fabric density. We have shown how the fabric density would affect the Lorentz transformation and we have provided a derivation from a particle basis. Fabric density is expected to show temporal variation with the evolution epoch of the universe, and spatial variation across aggregations of matter.

If this is correct, then several cosmological phenomena will need reinterpretation. The fabric effect is covert in that it causes remote particles to change frequency and velocity in ways that are non-obvious to observers. Consequently, it is possible that human observers may be attributing other mechanisms to effects that could be due to anisotropic fabric density. Many cosmological phenomena are formulated with the Lorentz or the conventional red-shift. These effects include the Hubble expansion, dark energy, galaxy rotation curves, dark matter. If the red-shift has a dependency on fabric density as we propose then a re-interpretation of these effects will be necessary.

\subsection{Outcomes}

Using particles in the derivation is not novel per se. The originality is achieving this using NLHV theory. Specifically, we have shown that is it possible to derive the Lorentz transformation, relativistic time dilation, and relativistic Doppler, i.e. key features of relativity can be derived using this NLHV theory.

Another contribution is that we propose an enlargement of the Lorentz formulation to include a new cosmological variable of fabric density. This variable does not appear in other theories, and is a falsifiable prediction of the Cordus Theory. This is a potentially significant development as it has multiple further implications, as discussed above. Hence, it is predicted that the conventional Lorentz is incomplete, that inertial frames of reference are only situationally equivalent in the special case where they also have the same fabric density, and that the speed of light is only constant within an isotropic fabric. We propose instead that the speed of light depends on the fabric density. We have derived the Lorentz under this more general set of assumptions, and also recovered the conventional Lorentz.

A wider contribution is that the paper provides a relativistic theoretical component which contributes to the development of the NLHV sector. In particular, the present work provides a quantitative formulation that supports the earlier conceptual work on time as an emergent property of matter [21], the nature of the vacuum [22], and the origin of the finite speed of light [23].

A philosophical contribution is providing an explanation for relativity based on a deeper physical realism. Specifically the Cordus Theory proposes physical structures at the sub-particle level, and offers a set of principles to govern the deeper mechanics. Curiously, these mechanisms are deterministic, but there are 
hints that at a coarser resolution the effects manifest as stochastic behaviours. This is consistent with wave function of QM. Hence we see possibilities for a deeper theoretical compatibility between the Cordus Theory and both quantum mechanics and general relativity.

The key differentiating factor in the present Lorentz derivation is the proposed continuity of the flux tube, which is part of the sub-structure of the particule. The yet deeper mechanics of the flux tube is not elucidated by this theory. The implication is that there may be still deeper mechanics to explore. This contrasts with QM which does not allow inner structures except as intrinsic variables [27] [28].

\subsection{Implications for Future Research}

The accelerating expansion of the universe is conventionally attributed to dark energy, the nature of which is unknown. In contrast, our theory suggests the phenomenon may be due to epochal changes in fabric density. Specifically, the fabric density was greater in the early epoch of the universe, assuming an explosive release of matter at baryogenesis. In later epochs such as the present, the dilution of matter across space may have caused a reduced fabric density, causing massy bodies to experience an intrinsic increase in their velocity (per Equation (2.3)).

Within any one epoch, the fabric density would also change with spatial position in the universe, being greater around concentrations of mass. The Cordus Theory predicts that the lower fabric density in the distal galactic regions causes these distal stars to experience an intrinsic increase in velocity per Equation (2.3). Hence, we suggest that the gradient in fabric density contributes to the anomalous rotation curves of galaxies, such that dark matter may not be necessary in the quantities expected. Further work is required to quantify the effect of fabric density in rotation curves.

The wider implication of situational relativity is that velocity phenomena, such as cosmological expansion and galaxy rotation profiles, may be at least partly artefacts of temporally and spatially variable fabric density respectively.

\section{Conclusions}

We set out to prospect for a relativistic formulation from a particle perspective. We achieved this by deriving the Lorentz transformation using the Cordus Theory. We find that the fabric density, hence distribution of matter, is an essential component of the Lorentz formulation under these assumptions. We propose a new formulation of the Lorentz that includes the relative difference in fabric density between source and observer. In the case where there is no difference in fabric density, then the conventional Lorenz is recovered.

We predict that the underlying causality for relativistic effects includes not only velocity, but also the background fabric density. This is not anticipated by other theories, and is a falsifiable prediction. We propose that the concept of a 
"reference frame" in conventional relativity needs to be augmented with fabric density ("situation").

We extended this to derive equations for relativistic time dilation and Doppler. Obtaining the relativistic Doppler equation is significant, since it is a key feature of general relativity that has not previously been demonstrated from a particle basis.

This complements earlier work that provided a qualitative description of time dilation [21]. Hence we now have a more comprehensive theory for time. The theory describes how time arises at the fundamental level (via the emission of discrete forces from particules), how this phenomenon aggregates to cause the time we experience at the macroscopic level, why time is irreversible (also explaining the origin of entropy), and provides a formulation for how time dilation and relativistic effects operate [21] [20]. The resulting situational theory of relativity encompasses aspects of both relativity and particle interactions, hence provides an integration between wave theory, particle physics, and relativity.

\section{Author Contributions}

All authors contributed to the creation of the underlying concept, development of the ideas, and editing of the paper.

\section{Acknowledgements}

We thank Ariel Pons for contributing to earlier ideas on which the present work was built.

\section{Conflict of Interest statement}

The authors declare that there are no financial conflicts of interest regarding this work. The research was conducted without personal financial benefit from any third party funding body, nor did any such body influence the execution of the work.

\section{References}

[1] Einstein, A. (1920) Relativity: The Special and General Theory. Henry Holt and Company, New York.

[2] Ives, H.E. (1945) The London, Edinburgh, and Dublin Philosophical Magazine and Journal of Science, 36, 392-403.

[3] Jozsef, C. (2016) Journal of Modern Physics, 7, 952-963. https://doi.org/10.4236/jmp.2016.79087

[4] Field, J.H. (1997) Helvetica Physica Acta, 70, 542-564.

[5] Lévy-Leblond, J.-M. (1976) American Journal of Physics, 44, 271-277. https://doi.org/10.1119/1.10490

[6] de Broglie, L. (1925) Annales de Physique, 3, 3-109.

[7] Bohm, D. and Bub, J. (1966) Reviews of Modern Physics, 38, 453-469. https://doi.org/10.1103/RevModPhys.38.453 
[8] Ellis, B. (2005) Ratio, 18, 371-384. https://doi.org/10.1111/j.1467-9329.2005.00300.x

[9] Pons, D.J., et al. (2012) Physics Essays, 25, 132-140. https://doi.org/10.4006/0836-1398-25.1.132

[10] Pons, D.J. (2015) Internal Structure of the Electron (Image Licence Creative Commons Attribution 4.0). Wikimedia Commons, Creative Commons Attribution 4.0 International license.

[11] Pons, D.J., Pons, A.D. and Pons, A.J. (2014) Applied Physics Research, 6, 50-63.

[12] Pons, D.J., Pons, A.D. and Pons, A.J. (2015) Applied Physics Research, 7, 1-11. https://doi.org/10.5539/apr.v7n6p1

[13] Pons, D.J., Pons, A.D. and Pons, A.J. (2014) Applied Physics Research, 6, 28-46.

[14] Pons, D.J., Pons, A.D. and Pons, A.J. (2015) Journal of Nuclear and Particle Physics, 5, 58-69.

[15] Pons, D.J. (2015) Applied Physics Research, 7, 14-26.

[16] Pons, D.J., Pons, A.D. and Pons, A.J. (2016) Journal of Modern Physics, 7, 1049-1067. https://doi.org/10.4236/jmp.2016.710094

[17] Pons, D.J., Pons, A.D. and Pons, A.J. (2015) Physics Research International, 2015, 1-19 (Article ID 651361).

[18] Pons, D.J., Pons, A.D. and Pons, A.J. (2013) Applied Physics Research, 5, 145-174.

[19] Pons, D.J., Pons, A.D. and Pons, A.J. (2014) Journal of Modern Physics, 5, 1980-1994. https://doi.org/10.4236/jmp.2014.517193

[20] Pons, D.J., Pons, A.D. and Pons, A.J. (2016) Journal of Modern Physics, 7, 1277-1295. https://doi.org/10.4236/jmp.2016.710113

[21] Pons, D.J., Pons, A.D. and Pons, A.J. (2013) Applied Physics Research, 5, 23-47.

[22] Pons, D.J. and Pons, A.D. (2013) The Open Astronomy Journal, 6, 77-89. https://doi.org/10.2174/1874381101306010077

[23] Pons, D.J., Pons, A.D. and Pons, A.J. (2016) Applied Physics Research, 8, 111-121. https://doi.org/10.5539/apr.v8n3p111

[24] Pons, D.J., Pons, A.D. and Pons, A.J. (2013) Relativity and Cosmology, vixra: 1305.0148, 1-15. http://vixra.org/abs/1305.0148

[25] Fischer, E. (2017) International Journal of Astronomy and Astrophysics, 7, 303-312. https://doi.org/10.4236/ijaa.2017.74025

[26] Michelson, A.A. and Morley, E.W. (1887) American Journal of Science, 34, 333-345. https://doi.org/10.2475/ajs.s3-34.203.333

[27] Colbeck, R. and Renner, R. (2008) Physical Review Letters, 101, 050403. https://doi.org/10.1103/PhysRevLett.101.050403

[28] Colbeck, R. and Renner, R. (2011) Nature Communications, 2, Article Number: 411. https://doi.org/10.1038/ncomms1416 